\title{
Current perspectives in the USA on the diagnosis and treatment of pelvic inflammatory disease in adolescents
}

This article was published in the following Dove Press journal:

Adolescent Health, Medicine and Therapeutics

27 June 2017

Number of times this article has been viewed

\author{
William L Risser' \\ Jan M Risser ${ }^{2}$ \\ Amanda L Risser ${ }^{3}$ \\ 'Department of Pediatrics, University \\ of Texas Medical School, ${ }^{2}$ Division \\ of Epidemiology, University of Texas \\ School of Public Health, Houston, \\ TX, ${ }^{3}$ Department of Family Medicine, \\ Oregon Health and Sciences \\ University, Portland, OR, USA
}

Correspondence: William L Risser I33I Se 76th Ave, Portland, OR 972I5, USA

Tel +I 5032550150

Email wlrisser@gmail.com
Abstract: In this review, the epidemiology, diagnosis, and treatment of pelvic inflammatory disease (PID) are discussed from a USA perspective and the difficulties that USA adolescents face in recognizing and seeking care for PID and other sexually transmitted infections (STIs) are emphasized. Females aged 15-24 years have the highest incidence of cervical infection with Chlamydia trachomatis and Neisseria gonorrheae, the principal causes of PID. PID is common in this age group. However, the incidence of PID in the USA is not known, because it is not a reportable disease, and because clinicians vary in the criteria used for the diagnosis. The Centers for Disease Control and Prevention (CDC) recommended the following diagnostic criteria that include lower abdominal or pelvic pain and at least one of the following: adnexal tenderness or cervical motion tenderness or uterine tenderness. Because PID can have serious sequelae, the criteria emphasize sensitivity (few false-negatives) at the expense of specificity (some false-positives). Patients who have PID are usually treated in the outpatient setting, following the CDC's Guidelines for the Treatment of Sexually Transmitted Diseases 2015. They receive one dose of an intramuscular cephalosporin, together with 2 weeks of oral doxy cycline, and sometimes oral metronidazole. Improvement should usually be evident in 3 days. The USA does not offer comprehensive sex education for adolescents in public or private schools. Adolescents are unlikely to recognize the symptoms of PID and seek medical treatment. Confidentiality is important to adolescents, and low cost or free sources of confidential care are uncommon, making it unlikely that an adolescent would seek care even if she suspected an STI. The CDC has concluded that screening programs for chlamydia and gonorrhea infection help prevent PID; however, the lack of appropriate sources of care makes adolescents' participation in screening programs unlikely.

Keywords: pelvic inflammatory disease, diagnosis, treatment, prevention, adolescence, Chlamydia trachomatis, Neisseria gonorrheae, bacterial vaginosis, sex education, intrauterine device, epidemiology

\section{Plain language summary}

Pelvic inflammatory disease (PID) is an infection of the female reproductive organs. In general, females who have this infection are not very sick. However, the long-term complications such as infertility, chronic pelvic pain, and abnormal locations of pregnancies (ectopic pregnancy) are quite common after PID has resolved.

The Centers for Disease Control and Prevention (CDC) in the USA provides guidelines for the diagnosis and treatment of PID. It also advises that PID can be prevented in females if they are screened and treated for Chlamydia trachomatis, the most common germ that causes PID.

Most adolescents in the USA do not receive thorough sex education. Therefore, they are unlikely to know the symptoms of PID and why it should be treated. If they want treatment, 
they are unlikely to know of a clinical facility where they can obtain low-cost or free confidential care for possible PID and other sexually transmitted infections (STIs). They are also unlikely to know that screening for chlamydia infection is important, or to have a site where they can be screened. Reliable use of condoms can prevent the infections that cause PID, but adolescents often do not know the importance of condoms or how to use them.

\section{Background}

PID is a serious sexually acquired infection common in females aged $<25$ years. This review discusses the clinical aspects of PID and barriers adolescents face in obtaining care when ill and in participating in prevention programs. The review also discusses PID from the perspective of the USA; much of the information is applicable elsewhere.

\section{Epidemiology}

Accurate estimates of PID incidence are not available because, in the USA, PID is not a reportable disease; therefore, there are no surveillance data. Also, while the CDC has established clinical diagnostic criteria, clinicians variably use these. ${ }^{1-3}$

The primary cause of PID is infection with Chlamydia trachomatis (chlamydia) and/or Neisseria gonorrhoeae (gonorrhea) (more on the pathophysiology later). ${ }^{4}$ Both chlamydia and gonorrhea are reportable infections in the USA. CDC surveillance rates are calculated using reported cases identified at testing and underestimate the true prevalence of the infection because many infections go undiagnosed. ${ }^{5}$ The National Health and Nutrition Examination Survey (NHANES) collects the population prevalence of both infections through tests performed among a representative sample of the USA population and gives more precise prevalence estimates. $^{5}$

Females aged 15-24 years have the highest surveillance prevalence (positive diagnosis reported to the CDC) and the highest population prevalence (positivity at interview among a probability sample of the population) of both chlamydia and gonorrhea. ${ }^{6}$

\section{Chlamydia}

NHANES population prevalence of chlamydia in females aged $15-24$ years is $3.2 \%$ (95\% confidence interval [CI] $2.3 \%-4.5 \%) .^{7}$ Prevalence by race/ethnicity for females aged 14-24 years is $13.5 \%$ (95\% CI 9.2\%-17.7\%) among black females, 4.5\% (95\% CI 2.6\%-6.4\%) among Hispanic females, and 1.8\% (95\% CI $0.3 \%-3.2 \%)$ among white females. ${ }^{8}$
In 2015, females aged 15-24 years represented 47\% $(724,709 / 1,526,658)$ of all reported chlamydia cases (both male and female), yet they make up $7 \%$ of the total population. ${ }^{6,9}$ Chlamydia rates were 3.0 cases per 100 females for females aged 15-19 years and 3.7 cases per 100 females for females aged 20-24 years. Rates per 100,000 population by race/ethnicity are presented in Table $1 .^{6}$

\section{Gonorrhea}

NHANES population prevalence of gonorrhea among females aged $15-24$ years is $0.62 \%(95 \%$ CI $0.38 \%-1.03 \%))^{7}$ The population estimates of gonorrhea infection by race/ ethnicity among females aged 15-24 years were not reported. CDC reported the gonorrhea rates for 14- to 19-year-old females and 20- to 24-year-old females to be 0.44 and 0.55 per 100 females, respectively. Rates per 100,000 population by race/ethnicity are presented in Table 1.6

An estimated $10 \%-20 \%$ of females with chlamydia or gonorrhea cervicitis develop PID if the infection is not treated. ${ }^{4,10}$ In a small prospective study of adolescent females detained at a juvenile detention center, where the prevalence of chlamydia and/or gonorrhea was significantly higher $(19 \%)$ than in most groups of adolescents, the prevalence of PID at admission was $8.6 \%$ (95\% CI 5.7\%-12.2\%), and the incidence of PID during the first 31 days of incarceration was $7.9 \%$ (95\% CI 5.0\%-12.3\%). ${ }^{11}$

From NHANES data, the self-reported history of PID among females aged $18-44$ years was $4.4 \%$ (95\% CI $2.1 \%-$ $5.7 \%$ ) and among females aged 18-24 years, the self-reported history was $2.9 \%(95 \%$ CI $0.8 \%-5.0 \%){ }^{12}$

Risk factors for the development of PID are the same as for other STIs: age $<24$ years, multiple sex partners, use of non-barrier methods of contraception, douching, and low socioeconomic status. ${ }^{3}$

Table I Rates per 100,000 persons of chlamydia and gonorrhea, and rate ratios comparing black and Hispanic to white, among females aged 15-24 years

\begin{tabular}{lllll}
\hline & I5-19 years & Ratio & 20-24 years & Ratio \\
\hline Chlamydia & & & & \\
White & $1,339.1$ & 1.0 & $1,737.8$ & 1.0 \\
Black & $6,340.3$ & 4.7 & $6,782.5$ & 3.9 \\
Hispanic & $1,775.2$ & 1.3 & $2,500.9$ & 1.4 \\
Gonorrhea & & & & \\
White & 136.4 & 1.0 & 195.8 & 1.0 \\
Black & $1,547.3$ & 11.3 & $1,760.5$ & 9.0 \\
Hispanic & 191.5 & 9.0 & 263.0 & 1.3 \\
\hline
\end{tabular}

Notes: Data from CDC Surveillance, 2015.' Denominator data were not available and $95 \%$ confidence intervals around the calculated rate ratios could not be estimated. Abbreviation: CDC, Centers for Disease Control and Prevention. 


\section{Pathophysiology}

In general, PID presents with endometritis and salpingitis (mild-to-moderate disease) and occasionally with tubo-ovarian abscess or pelvic peritonitis (severe disease). ${ }^{2}$ PID should be thought of as a polymicrobial disease. The most common causative organism is chlamydia, followed by gonorrhea, and both may be present. Yet, these organisms account for only $50 \%$ of PID. ${ }^{1,13,14}$ Mixed infections caused by organisms in the vaginal flora, including the organisms that cause bacterial vaginosis, can cause PID $^{1,15}$ and can be associated with chlamydia and/or gonorrhea infections. ${ }^{1}$ Also implicated are cytomegalovirus, Mycoplasma hominis, Ureaplasma urealyticum, and Mycoplasma genitalium. ${ }^{1}$

While the acute infection of PID is usually not serious, the long-term sequelae are. In one report, women with a history of PID developed tubal scarring that leads to infertility in $8 \%$, ectopic pregnancy in $9 \%$, and chronic pelvic pain in $18 \% .{ }^{16}$ As many as $85 \%$ of women with presumptive PID do not seek prompt medical care, because of the non-severe, non-specific PID symptoms. ${ }^{17}$ Delays in diagnosis and treatment of PID are a risk factor for impaired fertility. ${ }^{17}$

\section{Clinical presentation}

The hallmark of the presentation of PID is lower abdominal or pelvic pain, generally bilateral. ${ }^{4}$ The pain usually is not severe, is dull in character, and has a slow onset. ${ }^{4}$

In a report of 117 consecutive adolescents diagnosed with PID, 102 (87\%) had mild disease (pain without other symptoms); 15 (13\%) had moderately severe PID (constitutional symptoms including fever, nausea, and/or vomiting); and none had generalized peritonitis or tubo-ovarian abscess (severe disease). ${ }^{18}$

Other possible complaints in adolescents who have PID include prolonged or increased menstrual bleeding; dysmenorrhea that is more painful, has lasted longer, or is different in quality than usual; dysuria resulting from associated urethritis; dyspareunia; and increased vaginal discharge. ${ }^{3}$ PID often develops around the time of the menses. ${ }^{4}$

Although some cases of PID are asymptomatic, others are not diagnosed because the patient or the health care provider fails to recognize that PID is possible. Unfortunately, adolescents and older women with mild or asymptomatic PID may still be at risk for infertility. ${ }^{19}$

\section{Diagnostic criteria}

The following recommendations for diagnosing PID are intended to help health care providers recognize when PID is possible and to make sure that treatment is initiated promptly. ${ }^{1}$
These criteria emphasize sensitivity rather than specificity so that as many cases as possible are identified and treated, even if some non-cases are treated. ${ }^{1}$ Note that the diagnosis is based solely on the pain history and the bimanual pelvic examination.

Lower abdominal or pelvic pain and any one of the following:

1. Adnexal tenderness, or

2. Cervical motion tenderness, or

3. Uterine tenderness.

Patients may have other clinical findings suggesting PID, including abnormal bleeding, dyspareunia, vaginal discharge, and fever or other constitutional symptoms. Their presence may increase the specificity of the diagnosis but insisting on their presence will decrease the sensitivity. ${ }^{1}$

Ectopic pregnancy and appendicitis are the most serious problems in the differential diagnosis of PID; adolescents with possible PID should have a urine pregnancy test. Other possible diagnoses include constipation, gastroenteritis, cystitis, renal colic, and complications of an ovarian cyst, including rupture, bleeding, and torsion (rare in adolescents). Most of these have characteristic histories, symptoms, and signs and will not be associated with a positive bimanual pelvic examination. ${ }^{3}$

It may be difficult to obtain a history from an adolescent. She needs to be assured that your discussion with her is confidential and that you will not share the information with others without her permission. You should spend time alone with her while eliciting the history and performing the examination, although she may be more comfortable if a female, even if the clinician is female is also present. During the history, she may not reveal that she has lower abdominal pain until you elicit lower abdominal tenderness on examination. To learn about possible increased vaginal discharge, the clinician can ask if she had noticed more staining of her underwear. During the history and examination, clinicians need to avoid medical terms that adolescents probably will not understand, for example "vagina" and "uterus," or at least check the adolescent's understanding of these terms. ${ }^{3}$

An adolescent may complain of pain with any of the manipulations during the bimanual pelvic examination, particularly if this is her first exam. It can be helpful to ask her if her discomfort is pain or pressure. ${ }^{3}$

Infectious perihepatitis, or Fitz-Hugh-Curtis syndrome (FHCS), is a complication of PID resulting in infection of the anterior surface of the liver and the adjacent parietal peritoneum. It is usually associated with chlamydia and/or 
gonorrhea infection. It results in pain in the right upper quadrant pain (RUQ) that may radiate to the right shoulder. ${ }^{18}$ The pain may be associated with constitutional symptoms and elevated acute-phase reactants. Liver enzymes may be normal or slightly elevated. ${ }^{18}$ This infection may occur with or after salpingitis. The patient should be asked about a current or recent history of lower abdominal or pelvic pain, and a bimanual pelvic examination should be performed to help to determine if PID is present. ${ }^{18}$

In a study of 117 consecutive adolescents with PID, 5 (4.3\%, 95\% CI 1.4\%-9.7\%) had RUQ pain that improved during treatment for PID. It was felt that this clinical improvement with PID therapy indicated that the RUQ pain was caused by FHCS. One patient had constitutional symptoms. During the period of the study, no patient had isolated RUQ pain without symptoms of PID.

Clinicians should include perihepatitis in the differential diagnosis of acute upper right quadrant pain in sexually active females. ${ }^{18,20}$

\section{Therapy}

Patients who are diagnosed with PID and who are not seriously ill should be treated in an outpatient setting. Outpatient therapy is as effective as inpatient therapy for mild-tomoderate disease (if the patient takes her oral medication). ${ }^{13}$

Treatment for PID is thoroughly discussed in the CDC's Sexually Transmitted Disease Treatment Guidelines 2015, available without charge online. ${ }^{21}$ These guidelines are updated about every 3 years. The CDC on its website, and in the periodical Morbidity and Mortality Weekly Report (MMWR), provides important new information between updates, for example, changes in the antibiotic susceptibility of pathogens.

The CDC's treatment recommendations for outpatient therapy are one dose of a parenteral cephalosporin, followed by 2 weeks of oral doxy cycline, with or without 2 weeks of metronidazole. Small studies suggest that other outpatient regimens may be successful. ${ }^{1}$ These are discussed in the CDC Guidelines. ${ }^{1}$

This therapy should continue even if the tests for chlamydia and/or gonorrhea are negative. ${ }^{1}$

\section{Further management}

Adolescents should have decreasing symptoms (eg, a decrease in abdominal tenderness and reduction in uterine, adnexal, and cervical motion tenderness) within 3 days after initiation of therapy. ${ }^{1}$ The possible causes of lack of clinical improvement are noncompliance with therapy, the need for broadening the antibiotic coverage, the occurrence of tuboovarian abscess, and the wrong diagnosis. If the patient is taking her doxy cycline and the diagnosis of PID still seems to be correct, metronidazole may be added to her antibiotic regimen to provide better coverage for a mixed infection by aerobes and anaerobes. If the patient's symptoms are worse, hospitalization should be considered. ${ }^{1}$

The CDC's criteria for hospitalization include pregnancy; failure to comply with or tolerate an oral outpatient regimen; inability to exclude a surgical emergency such as appendicitis; worsening symptoms at the 48- to 72-hour follow-up; vomiting; high fever or other signs and symptoms suggesting severe illness; a known tubo-ovarian abscess; or evidence of peritonitis. ${ }^{1}$ Adolescents should abstain from sexual intercourse until therapy is completed and sex partners have been treated. The patients should be tested for chlamydia, gonorrhea, syphilis, and HIV. Nucleic acid amplification tests should be used for chlamydia and gonorrhea; first-catch urine or a vaginal swab are appropriate sample sources. ${ }^{1,3}$

Contraception should be discussed. The adolescent should be counseled about PID and other STIs and about the importance of condoms in disease prevention (and how to use them). ${ }^{1}$

All patients who have received a diagnosis of chlamydial or gonococcal PID should be retested 3 months after treatment. ${ }^{22}$ If retesting at 3 months is not possible, they should be retested as soon as possible.

Men who have had sex with an adolescent who has PID during the 60 days before her illness should be presumptively treated for chlamydia and gonorrhea, even if these organisms were not isolated from the woman. ${ }^{1}$ If her last sexual intercourse was $>60$ days before onset of symptoms or diagnosis, the most recent sex partner should be treated.

PID may develop between the time of testing and the time treatment is initiated for positive chlamydia and gonorrhea tests. At the Harris County Juvenile Detention Center (Houston, TX, USA), 13/99 (13\%) adolescents who had a positive nucleic acid amplification test for chlamydia and/or gonorrhea developed PID while awaiting the test results. ${ }^{11}$ These tests were performed in batches at the Houston Health Department; the mean time between collection of the tests and the reporting of results was 7.5 days (range 2-17 days). ${ }^{11}$

Although bacterial vaginosis is associated with PID, whether the incidence of PID can be reduced by identifying and treating women with bacterial vaginosis is unclear. ${ }^{15,23}$ 


\section{Intrauterine devices (IUDs) and PID}

An IUD is an appropriate type of contraception for an adolescent. ${ }^{24}$ The Society of Obstetricians and Gynaecologists of Canada (SOGC) notes that adolescents often have little knowledge or negative beliefs about IUD use. ${ }^{24}$ Whitaker et al found that a 3-minute counseling session about IUDs was effective in promoting their use in adolescents. ${ }^{25}$

Adolescents should be evaluated for symptomatic cervicitis and PID when they present for IUD placement. If they have either condition, they should have appropriate testing and treatment and IUD insertion should be delayed. ${ }^{24,26}$

If they have no symptoms of a STI, they should be screened for chlamydia and gonorrhea by using nucleic acid amplification tests, and syphilis and HIV. ${ }^{24,26}$ The IUD can be inserted on the same day as testing. In fact, same-day insertion is recommended so that insertion is not delayed, because adolescents may not return for another visit. Use of prophylactic antibiotics at the time of insertion has not been found to be useful in preventing PID. ${ }^{24,26}$

If an adolescent needs treatment for either symptomatic or asymptomatic chlamydia or gonorrhea, an effort should be made to treat her partner(s). ${ }^{24,26}$

In a large study of $>20,000$ women, 5,000 of whom were between the ages of 15 and 24 years, the rate of PID infection was elevated only during the 20 days immediately following IUD placement. The rate of PID infection ( 9.7 infections per 1,000 women-years) during the first 20 days after IUD insertion was 6.9 times the rate after the first 20 days (1.4 PID infections per 1,000 women-years), but the absolute risk was small (8.3 additional infections over 1,000 women-years). The rate of PID across the study, among women aged 15-24 years, was 3.48 per 1,000 person-years; this rate is 3.03 ( $95 \%$ CI 1.89-4.83) times greater than the rate for those $>24$ years (1.15 per 1,000 women-years). ${ }^{27}$

The CDC recommends the following for females who have an IUD in place and develop PID: ${ }^{1}$

1. They should be treated for PID by using the usual treatments;

2. The IUD does not need to be removed immediately;

3. The patient should be reassessed in 48-72 hours. If she is not improving clinically, removal of the IUD should be considered.

The CDC has concluded from the available evidence that treatment outcomes do not differ between women who continue or discontinue IUD placement. ${ }^{21}$ The SOGC recommends reevaluation after 72 hours in mild-to-moderate disease; in severe disease, clinicians can consider removing the device after antibiotics have been started. ${ }^{24}$

If the woman wants to discontinue IUD use, it should be removed after antibiotics have been started, to avoid the risk of bacterial spread that results from the removal..$^{24,26}$ Use of emergency contraception should be considered if this is appropriate. She should be offered another form of contraception. ${ }^{24,26}$

Much of the research concerning IUDs and PID has concerned non-hormonal devices. However, the CDC and the SOGC extend the above recommendations to both nonhormonal and hormonal IUDs. ${ }^{24,26}$

\section{Prevention of PID}

Much of the literature on disease screening to prevent PID concerns chlamydia, because chlamydia infection is 10 times more common than gonorrhea ${ }^{28}$ Several reviewers have evaluated the evidence that screening for chlamydia can prevent PID. After reviewing the four available clinical trials, Gottlieb et al concluded that "screening and treatment [for chlamydia] is an important and useful intervention to reduce the risk of PID among young women... although the magnitude of their contribution remains unclear. ${ }^{29}$ However, two of these trials discussed by Gottlieb et al did not find significant differences in PID incidence between screened and unscreened subjects, and all had methodologic flaws. The evidence for the efficacy of screening in this review is not compelling.

In a comprehensive review, Price et al used mathematical modeling to estimate the risk of PID following a chlamydia infection and the proportion of chlamydia-related PID infections that could be prevented by screening and treatment. They conclude that the risk of diagnosed PID in women infected with chlamydia is $14.8 \%$ (95\% CI 4.8\%-24.8\%), based on the trial evidence from prospective studies. They also estimate that the risk of all PID, diagnosed and undiagnosed, is $17.1 \%(95 \% \mathrm{Cl} 5.6 \%-28.9 \%)$ and that $61 \%(95 \%$ $\mathrm{Cl} 55 \%-67 \%$ ) of chlamydia-related PID could be prevented by annual screening and treatment. ${ }^{30}$ The authors deal with problems they identify in the clinical trials and observational studies of the efficacy of screening and feel that their estimates of the effectiveness of screening are reasonable.

Zaher et al performed a systematic review to guide the recommendations of the US Preventative Services Task Force (USPSTF). They note that one trial did not find a significant reduction in the incidence of PID in the year following screening and treatment for chlamydia, whereas another found a benefit. As do the other reviewers, they regret the dearth of relevant studies of screening benefits and harms. ${ }^{31}$ 
In summary, the evidence that screening for chlamydia can prevent PID is not as compelling as most evaluators would like. However, many of the organizations that guide health care in the USA recommend screening, concluding that enough evidence exists to support this approach to PID prevention.

For example, in their most recent guideline, the USPSTF recommends screening sexually active women aged $\leq 24$ years for chlamydia and gonorrhea by using a nucleic acid amplification test for screening and single-dose therapy as treatment. They suggest that pregnant women who have positive tests should be retested 3 weeks after treatment. ${ }^{28}$

Other organizations that recommend chlamydia and gonorrhea screening (cited in the above USPSTF reference) are:

1. The CDC recommends annual screening for chlamydia and gonorrhea in all sexually active females aged $\leq 25$ years and in older women with specific risk factors. Because of the high likelihood of reinfection, the CDC also recommends retesting all patients diagnosed with chlamydial or gonococcal infections 3 months after treatment, regardless of whether they believe their partners have been treated.

2. The American Congress of Obstetricians and Gynecologists recommends screening for chlamydia and gonorrhea in sexually active females aged $\leq 25$ years and in women $>25$ years who have risk factors.

3. The American Academy of Pediatrics recommends routine annual screening for chlamydia and gonorrhea in all sexually active females aged $\leq 25$ years. It also recommends screening adolescents and young adults who have been exposed to chlamydia or gonorrhea in the past 60 days from an infected partner.

4. The American Academy of Family Physicians recommends screening for chlamydia and gonorrhea in sexually active females aged $\leq 24$ years and in older women who are at increased risk for infection.

5. Canadian guidelines recommend screening for chlamydia in all sexually active males and females $<25$ years and retesting at 6 months after treatment in infected patients.

Women must understand why screening is important, because they are unlikely to be symptomatic if they have chlamydia or gonococcal cervicitis: $80 \%-90 \%$ of chlamydia infections are asymptomatic, as are $\sim 80 \%$ of gonococcal infections. $^{20,32}$

\section{Adolescents and PID}

If an adolescent is to seek care for possible PID, the following must happen:
1. She must know that she can get infections from having sex.

2. She must know that she personally is at risk for such infections.

3. She must know that the symptoms that she has are consistent with one of those infections, namely PID.

4. She must know that she can be evaluated and treated for an STI without parental permission.

5. She must know where she can get confidential care that she can afford.

6. She must be able to buy the outpatient medicine.

7. She must be able to return to the clinician for follow-up.

8. She must be able to refer her sexual partner(s) for testing and treatment, or else discontinue having sex with him/ them.

Issues 1-3 as listed above: In the USA, 22 of 50 states and the District of Columbia (DC) mandate sex and HIV education. In all programs, 37 states require that sex education includes abstinence. Twenty-six of these require that abstinence be stressed. Thirteen states require that the information presented be medically accurate. Eighty-eight percent of schools allow parents to exclude their children from sexual health education. ${ }^{33}$

Seventy percent of female adolescents report talking to their parents about at least one of six sexual education topics that include STIs. Parental information is not always accurate. ${ }^{33}$ Clinicians may not talk with their adolescent patients about sexual education, and, if they do, the conversations are usually brief. ${ }^{33}$

Fifty-five percent of 7-12 graders have looked up sexual health information on the Internet, but the Internet information is often inaccurate. ${ }^{33}$

Therefore, it is unlikely that an adolescent, particularly a young adolescent, receives enough information to realize that she might have PID.

Cuffe et al found in their analysis of a national survey of youth aged 15-25 that, of the sexes combined, $41.8 \%$ felt that they were not at risk for STIs. ${ }^{34}$

All 50 states and the DC permit adolescents to seek diagnosis and treatment for STIs without parental consent. Some states allow the medical provider to notify parents or guardians if this is in the best interest of the adolescent. ${ }^{35}$ Confidentiality concerning their health information is important to many adolescents, and they may not seek care if they are not sure that confidentiality will be maintained. Cuffe et al also addressed this issue and found that $60.1 \%$ of males and $39.9 \%$ of females did not seek STI screening because of concerns about confidentiality. ${ }^{34}$ 
If the adolescent sees her private physician using the family medical insurance, the insurance company may issue an Explanation of Benefits to the family that includes diagnoses and tests. Adolescents may not know this, but they may think that it is likely that the physician will notify her parents of her visit.

The following are possible sites for low-cost, confidential care: public STI clinics; Planned Parenthood clinics; and school-based clinics. Some school-based clinics do not provide STI care because of community preference; others allow parents to opt their children out of STI care. All three of these resources are less likely to be available in rural and small-town settings. In addition, adolescents must be aware of the existence of these facilities and have transportation to them. The adolescents need to know that the facilities will provide confidential care. ${ }^{33}$ The adolescents who are described here might know of one of these facilities, perhaps by word of mouth from friends.

\section{How an adolescent might prevent PID}

The simplest means for an adolescent girl to prevent PID, other than abstinence, is to use condoms reliably. Unfortunately, adolescents are unlikely to learn how to use condoms in health education class. In 2014, 35\% of public and private high school students were taught how to correctly use a condom; in middle school, $10 \%$ were taught condom use. ${ }^{33}$ Most adolescents learn about condoms from other sources. ${ }^{36}$

Many organizations recommend screening for chlamydia to reduce the incidence of PID. ${ }^{29}$ However, adolescents face the same obstacles in obtaining screening for STIs that they face in obtaining care for active infections.

\section{Conclusion}

The CDC has clear recommendations for the diagnosis and treatment of PID in the USA. These are described above. However, given the inadequate sex education that most USA adolescents receive, they are not likely to recognize the symptoms of PID or to understand that they need to seek care if the symptoms are present. If an adolescent knows to seek care, she will have difficulty finding an affordable clinic where she is confident that her medical information is confidential. The same obstacles make her participation unlikely in a screening program for chlamydia and gonorrhea. Her risk of PID would decrease if her partner used condoms; again, because of poor sex education, she may not know the importance of condoms or the mechanics of their proper use. In the current conservative political climate of some parts of the USA, it seems unlikely that any of the above problems will disappear.

\section{Disclosure}

The authors report no conflicts of interest in this work.

\section{References}

1. Centers for Disease Control and Prevention. Sexually transmitted diseases treatment guidelines. MMWR Recomm Rep. 2015; 64(No. RR-3):1-137.

2. Morris GC, Stewart CMW, Schoeman SA, Wilson JD. A cross-sectional study showing differences in the clinical diagnosis of pelvic inflammatory disease according to the experience of clinicians: implications for training and audit. Sex Transm Infect. 2014;14(90):445-451.

3. Cromwel PF, Risser WL, Bartot AT. Coping with pelvic inflammatory disease in adolescents. Contemp Pediatr. 2004;21(4):33.

4. Paavonen J, Westrom L, Eschenbach D. Pelvic inflammatory disease. In: Holmes KK, Sparling PF, Stamm WE, et al., editors. Sexually Transmitted Diseases. 4th ed. New York: McGraw-Hill; 2008:1017-1050.

5. Torrone EA, Papp JR, Weinstock HS. Prevalence of Chlamydia trachomatis genital infection among persons aged 14-39 years - United States, 2007-2012. MMWR Morb Mortal Wkly Rep. 2014;63(38):934-838.

6. Centers for Disease Control and Prevention, Sexually Transmitted Disease Surveillance; 2015. Atlanta: US Department of Health and Human Services.

7. Satterwhite CL, Torrone E, Meites E, et al. Sexually transmitted infections among US women and men: prevalence and incidence estimates, 2008. Sex Transm Infect. 2013;40(3):187-192.

8. Torrone EA, Johnson RE, Tian LH, Papp JR, Datta SD, Weinstock H. Prevalence of Neisseria gonorrhoeae among persons 14 to 39 years of age, United States, 1999 to 2008. Sex Transm Dis. 2013;40(3):202-205.

9. United States Census Bureau; 2010. Available from: https://www.census. gov/2010census/data/. Accessed December 14, 2016.

10. Hook EWIII, Handsfield HH. Gonococcal infections in the adult. In: Holmes KK, Sparling PF, Stamm WE, et al., editors. Sexually Transmitted Diseases. 4th ed. New York: McGraw-Hill; 2008:627-645.

11. Risser WL, Risser JM, Benjamins LJ. Pelvic inflammatory disease in adolescents between the time of testing and treatment and after treatment for gonorrhoeal and chlamydial infection. Int J STDs AIDS. 2012;23(7): $457-458$.

12. Kreisel K, Torrone EA, Bernstein K, Hong J, Gorwitz R. Prevalence of pelvic inflammatory disease in sexually experience women of reproductive age - United States, 2013-2014. Morb Mortal Wkly Rep. 2017;66(3):80-83.

13. Ness RB, Soper DE, Holley RL, et al. Effectiveness of inpatient and outpatient treatment strategies for women with pelvic inflammatory disease: results from the Pelvic Inflammatory Disease Evaluation and Clinical Health (PEACH) randomized trial. Am J Obstet Gynecol. 2002;186(5):929-937.

14. Burnett AM, Anderson CP, Zwank MD. Laboratory-confirmed gonorrhea and/or chlamydia rates in clinically diagnosed pelvic inflammatory disease and cervicitis. Am J Emerg Med. 2012;30(7):1114-1117.

15. Ness RB, Kip KE, Hillier SL, et al. A cluster analysis of bacterial vaginosis-associated microflora and pelvic inflammatory disease. $\mathrm{Am}$ J Epidemiol. 2005;162(6):585-590.

16. Weström L, Joesoef R, Reynolds G, Hagdu A, Thompson SE. Pelvic inflammatory disease and infertility: a cohort study of 1,844 women with laparoscopically verified disease and 657 control women with normal laparoscopy. Sex Transm Dis. 1992;19(4):185-192.

17. Hillis SD, Joesoef R, Marchbanks PA, Wasserheit JN, Cates WJ, Westrom L. Delayed care of pelvic inflammatory disease as a risk factor for impaired fertility. Am J Obstet Gynecol. 1993;168(5): 1503-1509. 
18. Risser WL, Risser JM, Benjamins LJ, Feldman JM. Incidence of FitzHugh-Curtis syndrome in adolescents who had pelvic inflammatory disease. J Pediatr Adolesc Gynecol. 2007;20(3):179-180.

19. Wiesenfeld HC, Hillier SL, Meyn LA, Amortegui AJ, Sweet RL. Subclinical pelvic inflammatory disease and infertility. Obstet Gynecol. 2012;120(1):37-43.

20. Stamm WE. Chlamydia trachomatic infections of the adult. In: Holmes KK, Sparling PF, Stamm WE, et al., eds. Sexually Transmitted Dieases. 4th ed. New York: McGraw Hill; 2008:575-594.

21. Centers for Disease Control and Prevention. 2015 Sexually Transmitted Diseases Treatment Guidelines. Pelvic inflammatory disease (PID). Avaiable from: https://www.cdc.gov/std/tg2015/pid.htm. Accessed December 14, 2016.

22. Hosenfeld CB, Workowski KA, Berman S, et al. Repeat infection with chlamydia and gonorrhea among females: a systematic review of the literature. Sex Transm Dis. 2009;36(8):478-489.

23. Ness RB, Hillier SL, Kip KE, et al. Bacterial vaginosis and risk of pelvic inflammatory disease. Obstet Gynecol. 2004;104(4):761-769.

24. Caddy S, Yudin MH, Hakim J, Money DM. Best practices to minimize risk of infection with intrauterine device insertion. J Obstet Gynaecol Canada. 2014;36(3):266-274.

25. Whitaker AK, Terplan M, Gold MA, Johnson LM, Creinin M, Harwood B. Effect of a brief educational intervention on the attitudes of young women toward the intrauterine device. J Pediat Adolesc Gynecol. 2010;23(2): 116-120.

26. Curtis KM, Tepper NK, Jatlaoui TC, et al. U.S. Medical Eligibility Criteria for Contraceptive Use 2016. Morb Mortal Wkly Rep. 2016;65(RR-3): 1-104.

27. Farley TM, Rosenberg MJ, Rowe PJ, Chen JH, Meirik O. Intrauterine devices and pelvic inflammatory disease: an international perspective. Lancet. 1992;339(8796):785-788.
28. Final Recommendation Statement: Chlamydia and Gonorrhea Screening. U.S. Preventive Services Task Force; December 2016. Available from: https:/www.uspreventiveservicestaskforce.org/Page/Document/ RecommendationStatementFinal/chlamydia-and-gonorrhea-screening. Accessed March 3, 2017.

29. Gottlieb SL, Xu F, Brunham RC. Screening and treating Chlamydia trachomatis genital infection to prevent pelvic inflammatory disease: interpretation of findings from randomized controlled trials. Sex Transm Dis. 2013;40(2):97-102.

30. Price MJ, Ades AE, Soldan K, et al. The natural history of Chlamydia trachomatis infection in women: a multi-parameter evidence synthesis. Health Technol Assess. 2016;20(22):1-250.

31. Zaher B, Cantor AG, Pappas M, Daeges BA, Nelson HD. Screening for gonorrhea and chlamydia: a systematic review for the U.S. Preventive Services Task Force. Ann Intern Med. 2014;161(12):884-893.

32. Marrazzo JM, Handsfield HH, Sparling PF. Neisseria gonorrhoeae. Principles and Practice of Infectious Diseases. 7th ed. Philadelphia, PA: Churchill Livingstone; 2010

33. Guttmacher Institute. American Teens' Sources of Sexual Health Education; April 2016. Available from: https://www.guttmacher.org/factsheet/facts-american-teens-sources-information-about-sex. Accessed December 14, 2016

34. Cuffe KM, Newton-Levinson A, Gift TL, McFarlane M, Leichliter JS. Sexually transmitted infection testing among adolescents and young adults in the United States. J Adolesc Health. 2016;58(5):512-519.

35. Guttmacher Institute. An Overview of Minor's Consent Law; December 1, 2016. Available from: https://www.guttmacher.org/state-policy/ explore/overview-minors-consent-law. Accessed December 14, 2016.

36. Bortot AT, Risser WL, Cromwell PF. Condom use in incarcerated adolescent males: knowledge and practice. Sex Trans Dis. 2006;33(1): $2-4$.
Adolescent Health, Medicine and Therapeutics

\section{Publish your work in this journal}

Adolescent Health, Medicine and Therapeutics is an international, peer-reviewed, open access journal focusing on health, pathology, and treatment issues specific to the adolescent age group. All aspects of health maintenance, preventative measures and disease treatment interventions are addressed within the journal and practitioners from all disciplines are
Submit your manuscript here: http://www.dovepress.com/adolescent-health-medicine-and-therapeutics-journal

\section{Dovepress}

invited to submit their work as well as healthcare researchers and patient support groups. This journal is included in PubMed. The manuscript management system is completely online and includes a very quick and fair peer-review system. Visit http://www.dovepress.com/testimonials. $\mathrm{php}$ to read real quotes from published authors. 